\title{
Contribution à la connaissance de la dentition lactéale d'Ursus spelaeus
}

\author{
Par C. Rădulescu et P. SAmson
}

Avec 17 Figures

\begin{abstract}
$\mathrm{Z}$ us a mmen $\mathrm{f}$ assung: Das Studium von 68 Unterkiefern des $U$. spelaens, welche Exemplaren in einem Alter zwischen 6 Wochen und 16 Monaten angehören, erlaubt uns festzustellen, daß neben dem gewöhnlichen Fall mit d2, d3 und d4 auch Exemplare existieren, bei denen der d2 völlig rückgebildet ist. Der $\mathrm{d} 2$ ist - wenn er existiert - schräg eingepflanzt und zeigt eine markante morphologische Variabilität; seine Krone kann elliptisch- oder kreisförmig sein. Das Fehlen von Abwetzungsspuren und sein Auftreten in einer Fossette des Unterkiefers, nötigen uns zu der Annahme, daß dieser Zahn nicht gebraucht wird und in Rückbildung ist. Der d3 kann zwei distinkte Wurzeln haben, welche in einigen Fällen verschmelzen und mehr oder weniger deutliche Spuren dieses Prozesses zeigen; parallel dazu beobachtet man den $\mathrm{d} 3$ mit nur einer Wurzel. Der d4 ist ein sehr wechselhafter Zahn; neben dem Schema mit fünf Haupthöckern findet man eine wechselnde Zahl von Nebenhöckern. Die Reihenfolge, in welcher diese drei Milchzähne d2, d3 und d4 erscheinen, wia auch ihr Ausfallen, ist disto-mesial; der d2 kann bis zu 16 Monaten oder noch länger persistieren.
\end{abstract}

A bstract: The study of 68 mandibles of Ursus spelaeus, appertaining to individuals between 6 weeks and 16 months, allowed us to observe that besides the usual case with d2, d3, d4, there are specimens which have $\mathrm{d} 2$ in a completely reduced state; $\mathrm{d} 2$, when existing, is obliquely implanted and presents a marked morphological variability; its crown can be ellipsoidal or buttonlike; the lack of any marks of use and its insertion in a groove of the mandible allow us to infer that this tooth is not functional, but on its reduction way; $\mathrm{d} 3$ may present two distinct roots which in some cases join, having marks more or less evident of this process; in the same time we observe $\mathrm{d} 3$ with one root only; $\mathrm{d} 4$ is a tooth extremely variable; a variable number of accessory tubercles is added to the scheme with five principal cones. The order of appearance and shedding of the three milk-teeth, $\mathrm{d} 2, \mathrm{~d} 3, \mathrm{~d} 4$, is disto-mesial; $\mathrm{d} 2$ may persist till the age of 16 months and sometimes more.

L'existence d'énormes dépôts d'ossements de l'Ours des cavernes a produit, depuis longtemps, une littérature prodigieusement riche; pourtant, chose assez curieuse, les études sur les dents lactéales, restent encore à leur début. Bien que le matériel soit difficile à conserver, par sa nature même, les restes fossiles ne nous semblent pas être si rares comme on a déjà supposé.

Selon les informations que nous possédons, Nordmann (1858) est le premier auteur qui s'est occupé de la dentition lactéale chez les Ursidés (U.arctos et $U$.spelaeus). En ce qui concerne l'Ours des cavernes, NoRDMANn décrit antérieurement à la d41 ${ }^{1}$ ) deux ou trois alvéoles, assez variables quant aux distances et positions, excepté le plus distal, qui est toujours étroitement collé à la racine mésiale de la $\mathrm{d} 4$.

Des éclaircissements, les uns en partie valables, ont été apportés au même sujet par: Schlosser (1909), Kormos (1916), Pöhle (1923), Ehrenberg (1931), Friant (1933), Stehlin (1933), Mottl $(1934,1939)$.

En 1952, F.-Ed. Kову ${ }^{2}$ ) décrit pour la première fois toute la dentition lactéale d'U.spelaeus et analyse, en même temps, les travaux antérieurs, à l'exception de celui de Nordmann, sur lesquels nous ne reviendrons plus.

1) Nous préférons garder la nomenclature classique, en numérotant les dents jugales, tant définitives que lactéales, dans le sens mésio-distal $\left(P_{1}-P_{4}\right.$ et $\left.\mathrm{d} 2-\mathrm{d} 4\right)$. Les dimensions seront prises comme suit: hauteur sur la verticale, longueur dans le sens mésio-distal et largeur dans celui vestibulo-lingual.

2) Nous exprimons notre gratitude à M. lc docteur F.-Ed. KoBx, qui a eu l'amabilité de nous envoyer son ouvrage. 
A la suite des études mentionnées on a admis la suivante formule dentaire lactéale pour l'Ours des cavernes:

$$
\mathrm{id} \frac{3}{3} ; \mathrm{cd} \frac{1}{1} ; \mathrm{d} \frac{3}{3}
$$

Dans ce qui va suivre, nous nous bornerons à une esquisse des divers aspects de la réduction des dents de lait jugales, les seules qui subissent de fortes variations numériques et morphologiques.

Dans notre pays, malgré le nombre élevé des cavernes à l'ours, qui renferment d'immenses dépôts d'os, on n'a pas encore abordé l'étude de la première dentition d'U. spelaeus.

Nos recherches ont commencé pendant l'année 1952, quand l'un de nous a recueilli toute une série de pièces isolées dans la grotte "Colţul Surpat" (Région de Pitești). Ultérieurement en 1954, dans la grotte de Ferice (Région de Bihor) nous avons découvert avec M-elle E. Terzea, une très jeune $\mathrm{mandibule} \mathrm{munie} s \mathrm{e} u \mathrm{le} \mathrm{ment} d \mathrm{e} \mathrm{deux}$ dents jugales. Aussi, deux années plus tard, en compagnie de M-elle E. Terzea et de M. M. Ghika, une pièce semblable fut trouvée à l'occasion des fouilles exécutées par l'Institut de Spéologie "E. Racoviţă" dans la grotte "Colţul Surpat."3)

Le matériel que nous avons eu à notre disposition comporte 32 mandibules âgées de 6 semaines à 4 mois, 36 âgées de 9 à 16 mois et 30 autres appartenant à des individus adultes. L'âge a été estimé surtout d'après les données de PoHLE (l.c.), qui sont les plus complètes pour les Ours brun et polaire, avec la réserve qui nous est imposée par l'erreur inhérente à une comparaison entre les espèces disparues et actuelles. En ce qui concerne les dents jugales de la première dentition, quoiqu'elles ne soient pas toutes présentes sur la même mandibule, nous possédons au moins un exemplaire de chacune sur des mâchoires différentes. De nombreuses dents lactéales, à l'état isolé, spécialement la d4, ont été obtenues dans diverses grottes étudiées.

Le matériel que nous avons utilisé provient des stations suivantes: la grotte de Cioclovina (qui nous a fourni la plupart des pièces) et celle de Valea Drăcoasei (Région de Hunedoara), les grottes No. I et II de Valea Tătarului (Mts. Bucegi) et "Bordeiul de Piatră“ (Région de Constanţa).

La grotte de Cioclovina ${ }^{4}$ ) située à une altitude de $850 \mathrm{~m}$ contient un immense dépôt de restes fossiles d'U. spelaeus, qui s'étale sur une épaisseur de plus de $10 \mathrm{~m}$ et fut longtemps exploité dans un bût industriel. Dans cette grotte Roska MARTon (1912) a décrit l'existence de l'Aurignacien et du Moustérien, associés tout les deux aux débris d'Ours des cavernes. Nos recherches ont mis en évidence, dans la cadre faunistique, la présence de quelques nouveaux éléments représentés par: $U$. arctos (très rare), Canis lupus, Felis spelaea et Capra ibex.

Dans la grotte de Valea Drăcoasei, nos fouilles, sommaires d'ailleurs, ont abouti à la découverte, parmi des os provenant d'U.spelaeus, de quelques pièces dentaires lactéales appartenant à la même espèce.

Les grottes de Valea Tătarului ${ }^{5}$ ), à une altitude de $1548 \mathrm{~m}$, ont fourni des restes d'U.spelaeus, U.arctos (très rare), Canis lupus et Ibex priscus ${ }^{6}$ ), à côté d'une industrie moustérienne supérieure. La grotte no. II nous a livré une mandibule d'Ours brun fossile âgé de 4 mois.

Située dans le bassin de la Casimcea, à 25 m d'altitude, la grotte "Bordeiul de Piatră “7)

3) Les résultats de ces recherches ont été communiqués dans les séances de l'Inst. de Spéol. de septembre et décembre 1956.

4) Communication faite à la séance mensuelle de l'Inst. de Spéol. (mai, 1959).

5) Communication faite à la session scientifique de l'Inst. de Spéol. (10-12 mai 1958).

6) Cette dernière espèce citée par I. Maxim (Bul. Soc. St. Cluj, t. X. 1948).

7) M. Dumitresco, M. Ghika, C. Rădulesco, P. Samson, Peştera "Bordeiul de Piatră“ (Ghilingic) (á paraître). 
nous a témoigné des traces d'une habitation moustérienne supérieure avec une association faunistique composée de 24 espèces. ${ }^{8}$ )

Pour interpreter avec plus de certitude les diverses étapes de dévelopement des deux dentitions successives de l'Ours des cavernes, nous avons utilisé un matériel juvénile de Carnivores actuels (Canis, Vulpes, Meles, Lutra, Ursus arctos, Felis Ieo, Felis catus) et fossiles (Alopex, Crocuta spelaea, Ursus arctos, Felis spelaea). ${ }^{9}$ )

Pour interpréter avec plus de certitude les diverses étapes de développement des deux selon leurs affinités morphologiques et tant qu'il fut possible aussi selon leur degré de développement. Les pièces âgées de plus de 9 mois seront étudiées à part, pour nous permettre de relever la persistance, en certains cas, de la d2. Ainsi nous avons distingué les groupes suivants:

A.- Le groupe à trois dents jugales $(\mathrm{d} 2, \mathrm{~d} 3, \mathrm{~d} 4)$

$1^{\circ}$ la $\mathrm{d} 3$ avec deux racines;

$2^{\circ}$ la $\mathrm{d} 3$ avec deux racines soudées;

$3^{\circ}$ la $\mathrm{d} 3$ avec une racine.

B. - Le groupe à deux dents jugales $(\mathrm{d} 3, \mathrm{~d} 4)$

Parfois à ces groupes peut s'ajouter la $P_{1}$.

C.- - Le groupe à une seule dent jugale (d4).

\section{A.- Le groupe à trois dents jugales (d2, d3, d4)}

$1^{\circ}$. Parmi les pièces qui possèdent la $\mathrm{d} 3$ avec deux racines, nous avons choisi celles qui nous semblent être les plus significatives; ces mandibules sont âgées de 3 à 4 mois.

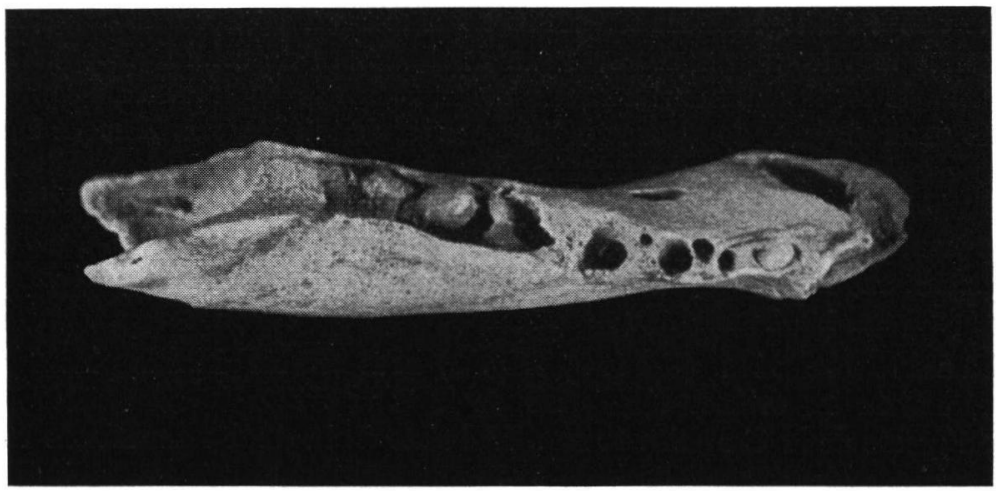

Fig. 1. U. spelaeus, mandibule dext. avec d2 (gr. nat.).

Sur la mandibule dext. (Cl. 563) (Fig. 1) on observe, en voie de se former, l'orifice d'éruption de la $\mathrm{C}$; cet orifice est situé tout près de l'alvéole de la $\mathrm{cd}$, dans sa partie linguale et approximativement à la moitié de son diamètre mésio-distal. Le germe de la $\mathrm{C}$, situé profondément dans la mandibule, a une longueur de $12.5 \mathrm{~mm}$. On remarque aussi l'orifice d'éruption de la $\mathrm{P}_{4}$, entre les deux alvéoles de la $\mathrm{d} 4$, vers la partie linguale; la $\mathrm{M}_{1}$ avec sa couronne déjà constituée est complètement à l'intérieur de la mandibule et présente une fente d'éruption longue de $20.8 \mathrm{~mm}$; la loge de la $\mathrm{M}_{2}$, dont le germe manque

8) Vespertilio serotinus, Myotis myotis, Mesocricetus auratus, Spalax leucodon, Microtus arvalis, M. nivalis, M. gregalis, M. oeconomus ratticeps, Lagurus lagurus, L.luteus, Spermophilus citelloides, Arvicola terrestris, Lagomys sp., Canis lupus, C. aureus?, Vulpes vulpes, Crocuta spelaea, Ursus spelaeus, Mustela sp., Equus caballus, E. caballus de grande taille, E. (Asinus) bydruntinus, Saiga tatarica, Bison priscus.

9) Nous devons de très vifs remerciements á M-me le Prof. Dr. M. Dumitresco pour le matériel de Mustélidés, à M. le Dr. T. Orghidan pour les photographies, à M. E. ŞERBan pour le matériel juvénile de Felis spelaea. 

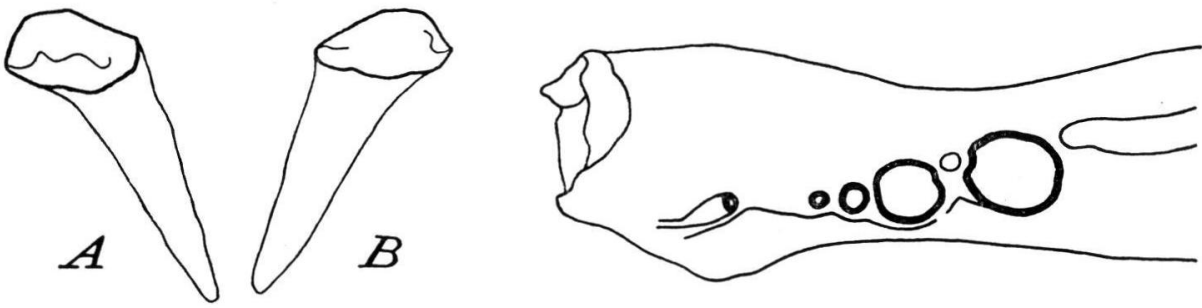

Fig. 2. U. spelaeus, la dent jugale antérieure (d2) A. partie linguale, B. partic vestibulaire ( $5 \mathrm{x}$ environ).

Fig. 3. U. spelaeus, mandibule sin. La ligne accentuée indique les alvéoles des dents lactéales $(1,5 \mathrm{x})$. sur notre matériel, forme une fente du côté lingual à la base de la branche ascendante mandibulaire.

La dent jugale antérieure (d2) (fig. 2), implantée de biais, a une seule racine complètement fermée et comporte une hauteur totale de $7 \mathrm{~mm}$. La couronne, penchée du côté lingual, plus longue que large $(3.2 \times 1.9 \mathrm{~mm})$, haute de $1.7 \mathrm{~mm}$, affecte une forme élipsoïdale. Une sorte de $\mathrm{c}$ in g u l u m est à remarquer surtout vers la partie mésio-linguale de cette dent. L'alvéole correspondant, ovale, avec le diamètre long de $3 \mathrm{~mm}$, est précédé par une fossette peu accentuée qui entoure sa partie mésiale et s'étend jusqu'à l'alvéole de la cd. Cette dent lactéale antérieure - à cause de la position oblique de la racine par rapport à la couronne et aussi à cause de la concavité mésiale qui se forme immédiatement sous la couronne - est étroitement collée à la partie distale de la fossette déjà mentionnée et en même temps est légèrement surplombée par le bord vestibulaire de la mandibule. Ce cas, comme tous les autres observés par nous, diffère de celui figuré par KoBY (1.c. Fig. 11) où la $\mathrm{d} 2$ est singulièrement proéminente. Après un intervalle d'un millimètre se trouvent les deux alvéoles de la dent jugale intermédiaire (d3), sensiblement égaux, avec un contour circulaire et situés obliquement dans le sens mésio-distal et vestibulo-lingual. Cette orientation est due au fait que l'alvéole distal, tout proche de l'alvéole mésial de la $\mathrm{d} 4$, est poussé vers le côté lingual, tandis que l'alvéole mésial reste dans l'axe longitudinal de la mandibule. Enfin, on peut observer les alvéoles (longueur $10.7 \mathrm{~mm}$ ) de la dernière dent jugale (d4) qui n'offrent rien de particulier.

L a mandibule sin. (Cl. 570) (Fig. 3) nous semble un peu plus âgée que la précédente, témoin les orifices d'éruption de la $\mathrm{C}$ et de la $\mathrm{P}_{4}$ mieux développés; la longueur du germe de la $\mathrm{C}$ atteint $15 \mathrm{~mm}$. L'alvéole de la $\mathrm{d} 2$, de dimensions réduites, se continue avec une échancrure longue de $5 \mathrm{~mm}$, orientée selon une direction distomésiale et linguo-vestibulaire, dans laquelle était logée probablement la couronne de cette dent. Par conséquent la position est contraire à celle de la jugale antérieure décrite plus haut. Les alvéoles de la $\mathrm{d} 3$, séparés de celui de la $\mathrm{d} 2$ par un espace de $4.5 \mathrm{~mm}$, ont la même orientation comme dans le cas précédent; on doit signaler seulement que l'alvéole mésial est aciculaire et peu marqué, tandis que le distal est rond et plus fort; la longueur des deux alvéoles comporte $4 \mathrm{~mm}$.

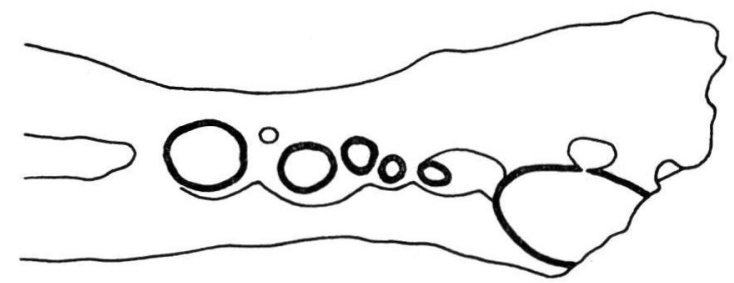

Fig. 4. U. spelaeus, mandibule dext. (1,5 x). 
Un état intermédiaire est représenté par la mandibule dext. (Cl. 560) (Fig. 4), dont l'alvéole de la $\mathrm{d} 2$ est circulaire avec un diamètre de $2.5 \mathrm{~mm}$ et les deux alvéoles de la d3, quoique inégaux, sont bien exprimés, ayànt une longueur de $4.8 \mathrm{~mm}$. La fossette antérieure de l'alvéole de la $\mathrm{d} 2$ est moins profonde que dans d'autres cas.

En général, lorsque la d 3 possède deux racines, la distale est plus forte que la mésiale.

$2^{\circ}$ Chez les mandibules à trois dents jugales, dont l'intermédiaire (d3) a les deux racines soudées, on peut observer les phases successives de ce processus. Les mandibules qui seront décrites sont âgées de 3 à 4 mois.

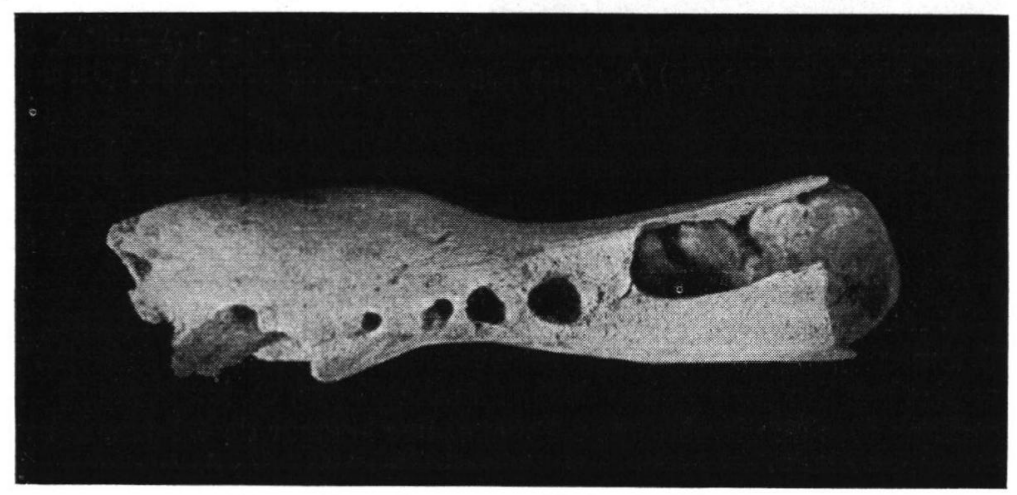

Fig. 5. U.spelaeus, mandibule sin. (gr. nat.)

Sur $1 \mathrm{a}$ mandibule sin. (Cl. 577) (Fig. 5), les orifices d'éruption de la $\mathrm{C}$ et de la $\mathrm{P}_{4}$ sont évidents; la $\mathrm{M}_{1}$ avec la couronne complètement formée est située encore à l'intérieur de la mandibule. L'alvéole de la d2, oblique, allongé dans le sens mésio-distal comporte une longueur de $3.5 \mathrm{~mm}$ et présente antérieurement la fossette que nous avons déjà mentionnée dans les cas précédents, mais laquelle sur cette pièce est moins exprimée. Après un espace de $3.2 \mathrm{~mm}$, suit l'alvéole de la $\mathrm{d} 3$ long de $4.2 \mathrm{~mm}$ présentant sur les parois vestibulaire et linguale deux $\mathrm{septums}$ qui avancent très peu vers le centre de l'alvéole dans sa partie supérieure et tendent à se joindre dans celle inférieure, indiquant ainsi la ligne de fusion des deux racines. On doit remarquer la position de cet alvéole dans l'axe longitudinal de la mandibule.

Sur un fragment de mandibule dext. (Cl. 574) on peut observer l'alvéole de la $\mathrm{d} 3$ dont le diamètre long prend une direction à peu près vestibulo-linguale; les s e p t u m s sont moins marqués.

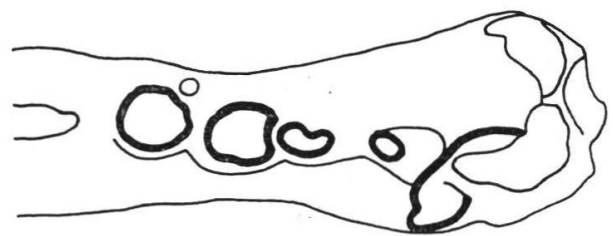

Fig. 6. U. spelaeus, mandibule dext. $(1,5 \mathrm{x})$.

La mandibule dext. (Cl. 575) (Fig. 6) laisse reconnaître un état plus avancé dans la fusion des racines de la $\mathrm{d} 3$; comme suite on remarque seulement une trace de se ptum sur la paroi linguale de l'alvéole.

Lorsque la dent jugale intermédiaire possède deux racines soudées, la partie mésiale de l'unique racine est plus courte et plus mince que la distale. 

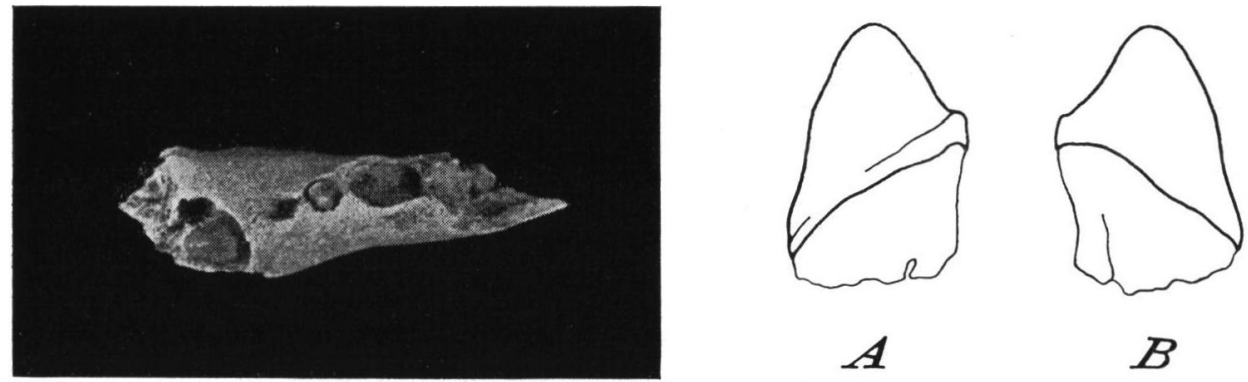

Fig. 7 (à droite). U.spelaeus, mandibule sin. avec $\mathrm{d} 3$ (gr. nat.). - Fig. 8 (à gauche). U. spelaeus, la dent jugale intermédiaire (d3) A. partie linguale, B. partie vestibulaire ( $5 \mathrm{x}$ environ).

$3^{\circ}$. La plupart du matériel que nous possédons, comporte des mandibules dont la $\mathrm{d} 3$ a une seule racine. Ces pièces proviennent de jeunes sujets âgés de 6 semaines à 4 mois.

La mandibule sin. (Cl. 579) (Fig. 7) provient d'un ourson qui n'a pas dépassé son deuxième mois (probablement 6 semaines). On peut observer la $\mathrm{cd}$ en voie d'éruption a vec sa pointe à $5 \mathrm{~mm}$ au-dessus du bord alvéolaire. $L a \mathrm{~d} 3$ avec sa couronne formée, située dans l'alvéole, fait éruption avec sa pointe. La cd a la couronne complètement constituée, haute de $9.3 \mathrm{~mm}$, avec une longueur de $8.6 \mathrm{~mm}$ et une largeur de $6 \mathrm{~mm}$ au collet. La racine, ébauchée, atteint une hauteur maxima de $8.7 \mathrm{~mm}$. La d3 (fig. 8), implantée de biais, a une couronne conique avec un diamètre de $3.2 \mathrm{~mm}$ et une hauteur de $4.2 \mathrm{~mm}$, la racine étant ouverte et longue à peine de $3 \mathrm{~mm}$ sur sa face mésiale. A la base de la couronne apparaît un bourrelet qui longe la dent dans sa partie linguale et s'efface vers son extrémité distale. L'alvéole de la cd a une longueur de $8.2 \mathrm{~mm}$. Le germe de la $\mathrm{C}$ à peine formé (hauteur = $6 \mathrm{~mm}$ ) est situé profondément dans la mandibule, sa loge ayant la tendence de s'ouvrir pour constituer l'orifice d'éruption.

L'alvéole de la $\mathrm{d} 2$, très ouvert, ovale, long de $4 \mathrm{~mm}$, contenait probablement le germe de la dent et se trouve étroitement collé à l'alvéole à peu près circulaíre (diamètre = $4.5 \mathrm{~mm}$ ) de la $\mathrm{d} 3$. Après un espace très réduit, suivent les alvéoles de la $\mathrm{d} 4$ incomplètement constitués, longs de $12.5 \mathrm{~mm}$. La dent, qui manque sur notre piècę, commençait à faire éruption et d'après la topographie des alvéoles ses racines étaient déjà ébauchées. Sous l'alvéole de la d4 s'observe la loge double de la $\mathrm{P}_{4}$ en voie de formation, oblique, orientée dans le sens mésio-distal et vestibulo-lingual, avec sa partie antérieure plus réduite que celle postérieure, configuration observable d'ailleurs sur des mandibules plus âgées lorsque la dent est déjà apparue. Selon la morphologie dentaire et alyéolaire de cette pièce, la plus jeune que nous avons découverte, il est à supposer que 1 'o r d r e d'ér u p tion des jugales est lesuivant: les deux dernières presque en
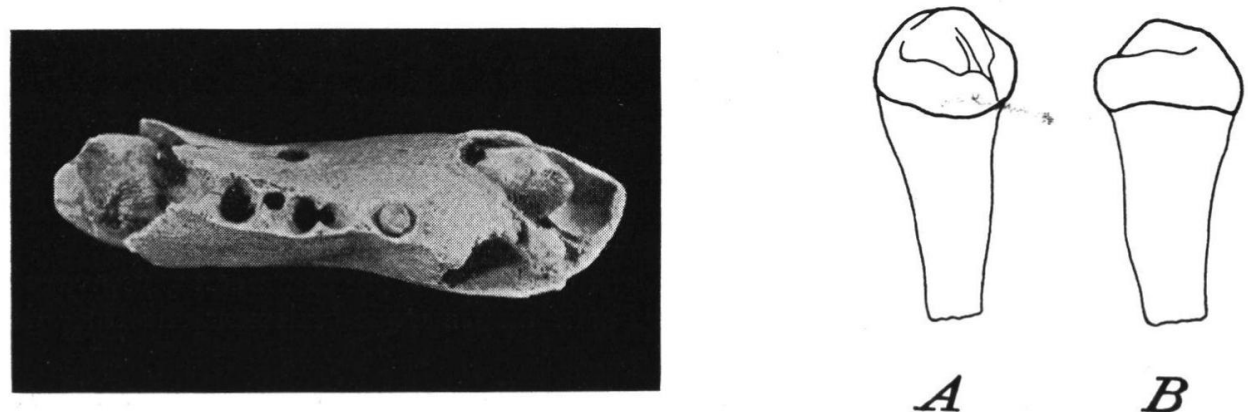

Fig. 9 (à droite). U. spelaeus, mandibul : dext. avec d2 (gr. nat.). - Fig. 10 (à gauche). U. spelaeus, la dent jugale antérieure (d2) A. partie vestibulaire, B. partie linguale ( $5 \mathrm{x}$ environ). 
même temps, la d4 étant cependant la première à paraître, succédées par la d 2 .

Sur une autre mandibule, dext. (Cl. 583) (Fig. 9), de presque 4 mois, la dent jugale antérieure (d2) est in $\mathrm{s}$ it u; l'alvéole de la d3 de même appliqué contre celui mésial de la d4; la distance entre les alvéoles de la d2 et d3 de $2.7 \mathrm{~mm}$. Dans ce cas, la d2 (Fig. 10) également implantée de biais, diffère de celle décrite par Koвy et par nous antérieurement à cause de sa couronne en bouton; la racine, continuant en ligne droite la couronne, est un peu ouverte et ainsi la hauteur totale de $6 \mathrm{~mm}$ reste approximative. Le diamètre mésio-distal de la couronne comporte $2.8 \mathrm{~mm}$, le transversal $2.5 \mathrm{~mm}$ et la hauteur $2.1 \mathrm{~mm}$. La couronne sans signes d'usure présente un cing u l u m discontinu situé dans la moitié mésio-vestibulaire de la dent. Dans la partie vestibulaire, l'interruption est marquée par un petit tubercule. L'alvéole de la $\mathrm{d} 2$, comme dans les cas précédemment décrits, forme une fossette antérieure, cependant n'atteignant pas l'alvéole de la cd.

Au même groupe se rattache la mandibule dext. (Cl. 586) (Fig. 11) dont la jugale postérieure ( $\mathrm{d} 4$ ) comporte $13.3 \mathrm{~mm}$ de longueur et $6.7 \mathrm{~mm}$ de largeur; l'âge de cette

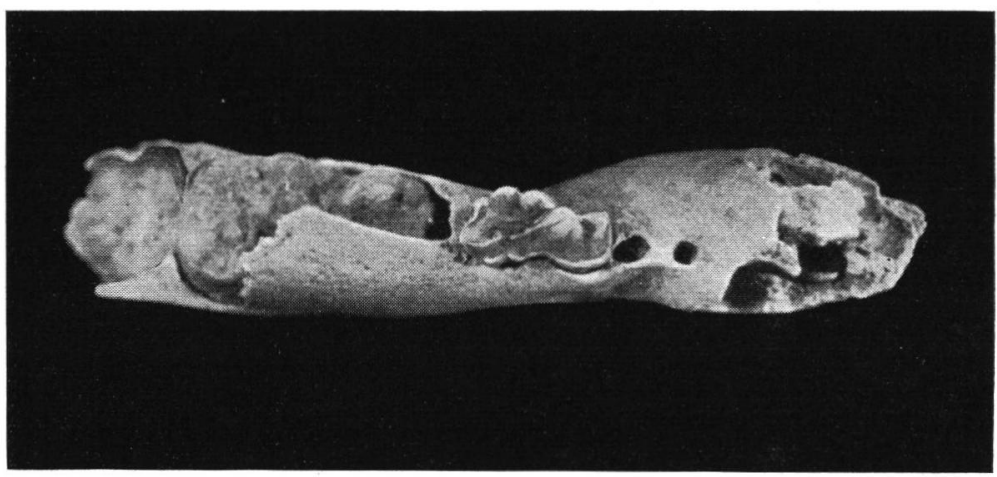

Fig. 11. U. spelaeus, mandibule dext. avec. d4 (gr. nat.).

pièce se place entre 3 et 4 mois. On connaît depuis longtemps les cinq cuspides principales de cette dent. En ce qui suit, nous nous proposons d'insister sur quelques variations propres à notre matériel, provenant de différentes grottes mentionnées, pour compléter les observations effectuées jusqu’à présent. Dans la partie mésio-vestibulaire le c ingulum puissamment développé peut individualiser un petit tubercule qui représente le point le plus antérieur de la dent. La crête distale du protoconide peut constituer dans sa moitié inférieure un ou deux tubercules, l'antérieur étant le plus fort; la partie vestibulaire du c ingulum a la possibilité de développer deux tubercules, le premier situé au niveau du sillon qui sépare le trigonide du talonide, le second à la base de l'hypoconide. On constate, dans certains cas, que le tubercule qui apparaît entre le para- et métaconide au lieu de rester indépendant se forme aux dépens de l'une ou de l'autre de ces cuspides. Parfois le métaconide présente aussi un petit tubercule dans sa partie disto-basale. Le c in g u $1 \mathrm{u} \mathrm{m}$ distal peut former encore deux tubercules placés chacun à la base de l'hypo- et de l'endoconide. En quelques cas un tubercule supplémentaire s'ajoute à celui de l'hypoconide. Dans le sillon qui sépare l'endo- et l'hypoconide, à la base de ce dernier, peuvent se développer jusqu'à trois tubercules de dimensions plus ou moins réduites.

Pour rendre plus évidente l'extrême complexité de la dernière jugale de l'Ours des cavernes, nous décrirons plus loin, la même dent appartenant à une $\mathrm{m}$ a $\mathrm{n} \mathrm{dibu} \mathrm{le} \mathrm{d}$ ex $\mathrm{t}$. (V.T.P. II. S.A. 1) (fig. 12) d'Ours brun fossile, dont l'âge ne dépasse pas le $4^{\mathrm{e}}$ mois. L'orifice d'éruption de la $\mathrm{C}$ se situe du côté lingual de l'alvéole de la cd, sa longueur représentant la moitié du diamètre longitudinal de ce dernier; on observe aussi l'orifice d'éruption de la $\mathrm{P}_{4}$; la $\mathrm{M}_{1}$ fait éruption avec ses pointes les plus élevées (proto- et méta- 


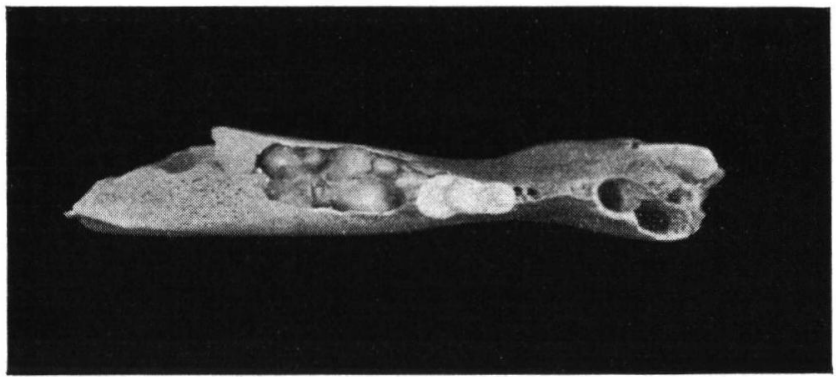

Fig. 12. $U$. arctos, mandibule dext. avec $\mathrm{d} 4$ (gr. nat. approx.).

conide). L'alvéole de la $\mathrm{P}_{1}$, séparé de la cd par un s e $\mathrm{t}$ u m interalvéolaire très mince, a $6 \mathrm{~mm}$ de longueur, $4.5 \mathrm{~mm}$ de largeur et une profondeur de $2.8 \mathrm{~mm}$. L'alvéole suivant, de forme aciculaire, appartient à la $\mathrm{d} 2$ et présente également une fossette antérieure qui entoure sa partie mésiale et s'étend cette fois-ci jusqu'à l'alvéole de la $\mathrm{P}_{1}$. Après un intervalle de $3.3 \mathrm{~mm}$ apparaissent deux alvéoles, le mésial ovale, le distal circulaire, pour la $\mathrm{d} 3$, ayant une longueur totale de $3.5 \mathrm{~mm}$. La d4 en comporte deux, dont le mésial est collé à celui distal de la d3. Chez l'Ours brun la d4 longue de $11.2 \mathrm{~mm}$ et large de $5.2 \mathrm{~mm}$, présente un paraconide plus détaché du protoconide à la différence de la configuration observée chez l'Ours des cavernes. Le c ingulum est à peu près absent dans la part mésio-vestibulaire de la dent et les tubercules font défaut. Entre le para- et métaconide peut s'insérer un petit tubercule; à la partie disto-basale du protoconide se forme également un autre tubercule. Le c ing u $1 \mathrm{u} \mathrm{m}$ se développe un peu plus dans la région distale du talonide et s'efface presque totalement vers celle vestibulaire; il constitue un tubercule à la base de l'hypoconide. L'endo- et l'hypoconide sont uniques, tandis que chez l'Ours des cavernes ils deviennent doubles ou même triples. Il est à signaler aussi un angle plus aigu entre le proto- et l'hypoconide chez l'Ours brun.

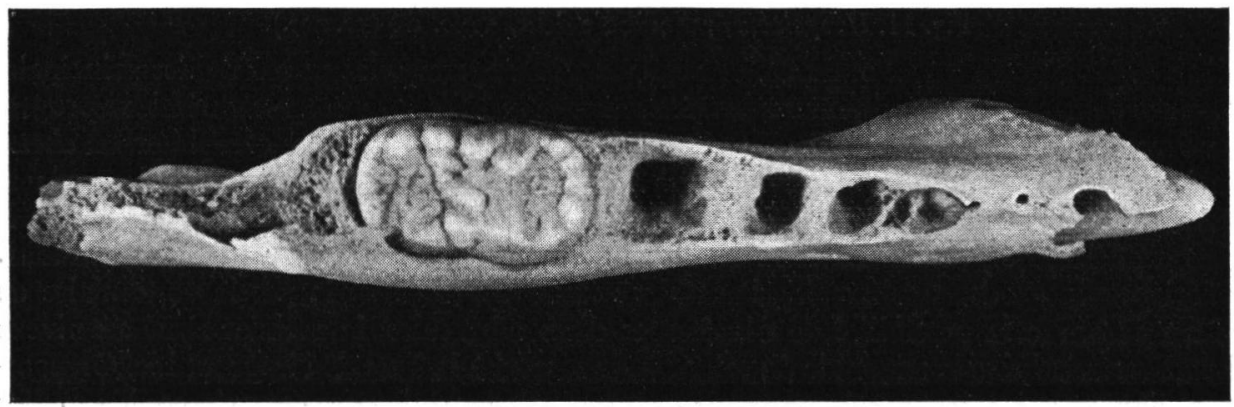

Fig. 13. U. spelaeus, mandibule dext. avec l'alvéole de la $\mathrm{P}_{1}(0,85 \mathrm{x})$.

Un cas particulier dénote $1 \mathrm{a}$ mandibule dext. (V.T.P. II. S1. I. 1) (Fig. 13) âgée d'environ 15 mois. Sur cette pièce est observable la $C$ qui a percé avec sa pointe. D'après les alvéoles de la $\mathrm{P}_{4}$ et de la $\mathrm{M}_{1}$ on peut déduire que ces dents ont fait déjà éruption; la $\mathrm{M}_{2}$ in situ est apparue jusqu'au collet dans sa partie mésiale, la moitié distale de la couronne étant encore enchassée dans l'alvéole; les racines sont encore ouvertes; la loge de la $\mathrm{M}_{3}$ est un peu oblique. On peut observer tout près de la $\mathrm{C}$ un alvéole postérieur, ovale, avec un diamètre longitudinal de $5.7 \mathrm{~mm}$ et une profondeur atteignant $9 \mathrm{~mm}$; cet alvéole d'après forme et position ne saurait appartenir qu'à la $P_{1}$. Après un espace de $5 \mathrm{~mm}$, il existe un alvéole correspondant à la d2, de petite taille et implantée de biais. Enfin, collé à l'alvéole de la racine mésiale de la $\mathrm{P}_{4}$ se trouve aussi l'alvéole de la $\mathrm{d} 3$, séparé de celui de la d2 par un intervalle de $3.7 \mathrm{~mm}$. 
La configuration, le nombre d'alvéoles et les dimensions plutôt faibles de cette pièce nous font penser à $U$.arctos. Toutefois la morphologie et les dimensions (longueur/largeur $=27.8 / 18.0$ ) de la $\mathrm{M}_{2}$ comparées avec un nombre élevé de dents tant d'U. spelaeus que d'U. arctos actuel et fossile nous détermine de l'attribuer avec plus de vraisemblance à un Ours des cavernes de petite taille, d'autant plus que ce fait est corroboré par l'existence parmi les pièces récoltées par nous d'une mandibule plus faible que la précédente, mais laquelle manifeste selon sa $\mathrm{P}_{4}$ des caractères nets de Spelaeus.

$$
\text { B.- Le groupe à deux dents jugales (d3 et d4) }
$$

Les mandibules de Ferice et de "Colţul Surpat" qui ont été déjà communiquées sont assez semblables à celles que nous allons décrire plus loin.

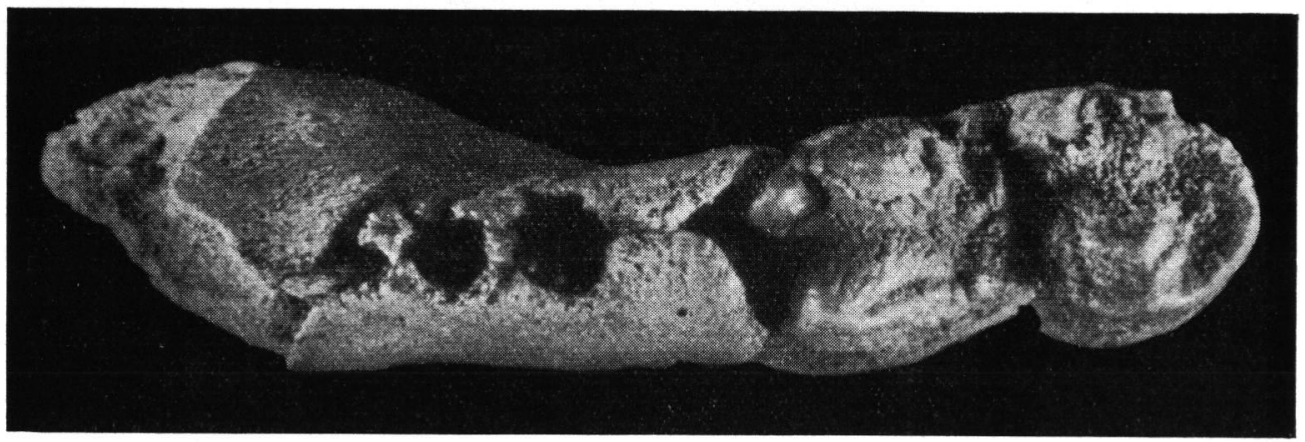

Fig. 14. U. spealets, mandibule sin. $(2 \mathrm{x})$.

L a mandibule sin. (V.T.P. II. S1. II. 2) (fig. 14), - dont l'orifice d'éruption de la $C$ n'est pas encore ouvert, la couronne de la $M_{1}$ et le germe de la $C$, long de $10 \mathrm{~mm}$, sont situés à l'intérieur de la mandibule - indique un ourson d'environ 3 mois. Tout ce qu'on peut observer dans ce cas, ce sont les deux alvéoles (longueur $=10,7 \mathrm{~mm}$ ) de la $\mathrm{d} 4$ auxquels s'ajoute, après un espace de $1.7 \mathrm{~mm}$, l'alvéole circulaire de la $\mathrm{d} 3$, avec un diamètre de $2.4 \mathrm{~mm}$; ce dernier est séparé de l'alvéole de la cd par une petite barre de $6.3 \mathrm{~mm}$.

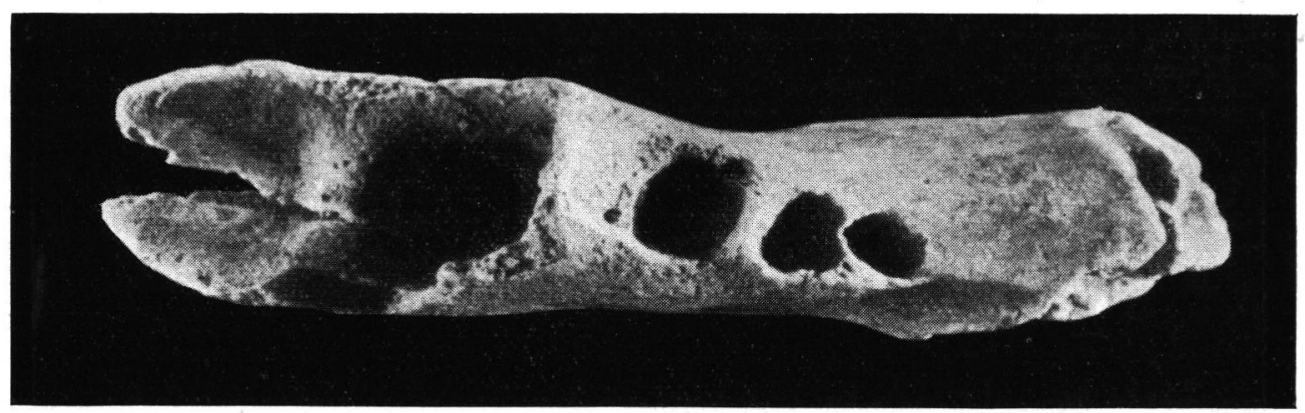

Fig. 15. U. spelaens, mandibule dext. (2x).

Sur une autre mandibule (Cl. 590) (Fig. 15), d'environ 4 mois, on voit les orifices d'éruption de la $\mathrm{C}$ et de la $\mathrm{P}_{4}$ et aussi, outre les deux alvéoles de la jugale postérieure, un autre, a peu près rond, d'une longueur de $4.7 \mathrm{~mm}$, appartenant à la $\mathrm{d} 3$; cet alvéole est séparé de celui de la cd par un espace de $6.3 \mathrm{~mm}$ et collé à celui mésial de la d4.

Sur une seule mandibule dext. (Cl. 589) (Fig. 16) âgée de 4 mois, avec les orifices d'éruption de la $\mathrm{C}$ et de la $\mathrm{P}_{4}$ constitués, nous avons constaté à côté des alvéoles de la 


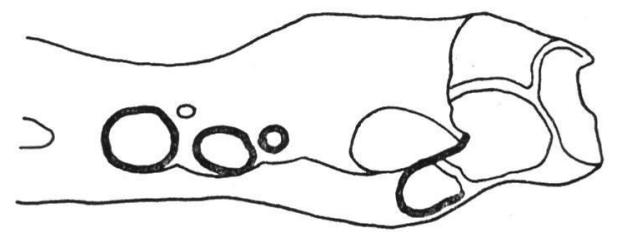

Fig. 16. U. spelaeus, mandibule dext. avec l'alvéole de la $P_{1}(1,5 \mathrm{x})$.

d4 et d3 l'existence d'un autre alvéole plus volumineux situé immédiatement à la partie distale de la cd; cet alvéole ne peut appartenir qu'au germe de la $\mathrm{P}_{1}$, selon ses dimensions qui comportent une longueur de $7.8 \mathrm{~mm}$ et une largeur de $4.8 \mathrm{~mm}$. L'alvéole de la jugale intermédiaire, ovale (longueur $2.6 \mathrm{~mm}$ ) est étroitement collé tant à l'alvéole mésial de la jugale postérieure qu'à celui de la $P_{1}$. Dans tous les cas de ce groupe, la jugale intermédiaire ( $\mathrm{d} 3$ ) ne possède qu'une seule racine, généralement implantée de biais.

$$
\text { C.- I. e groupe à une seule jugale (d4) }
$$

A propos de cette réduction très poussée, Schlosser (l.c.) remarque brièvement: „Zwischen der Alveole des CD und des D4 bemerken wir zwei kleine Alveolen, die offenbar dem D1 und D3 angehören. Sie fehlen fast stets an den jungen Bärenkiefern aus den fränkischen Höhlen“.

Nous avons eu l'occasion d'observer une configuration pareille sur une mandibule d'ourson trouvée dans le niveau moustérien de la grotte "La Adam" (Dobroudja). ${ }^{10}$ )

L'étude des mandibules dont l'âge oscille entre 9 et 16 mois, nous a permis de surprenćre les moments approximatifs d'expulsion des dents jugales lactéales.

En ce qui concerne ce processus Pohle (1.c.) admet pour les ours actuels l'ordre suivant: $\mathrm{d} 4$ à 10 mois, d3 à 13 mois environ, d2 à 9 mois. Koby (1.c.) se rapportant à la d2 affirme: "... que chez $U$. spelaeus cette dent est une des premières à tomber. Il en est de même d'ailleurs chez l'Ours brun, comme nous l'avons vérifié, et PoHLE a dû faire, pour ademettre cette persistance anormale, une confusion avec une prémolaire de la seconde dentition“. Pour l'Ours des cavernes, la mandibule Cl. 603 (Fig. 17) d'environ 14 mois, qui a la d2 in situ, nous semble être concluante. Par conséquent on peut admettre la justesse des observations de Pohle, d'autant plus que le cas ne semble pas être tellement isolé, vu que de 36 mandibules juvéniles examinées, 24 présentent l'alvéole de la $\mathrm{d} 2$ bien constitué.

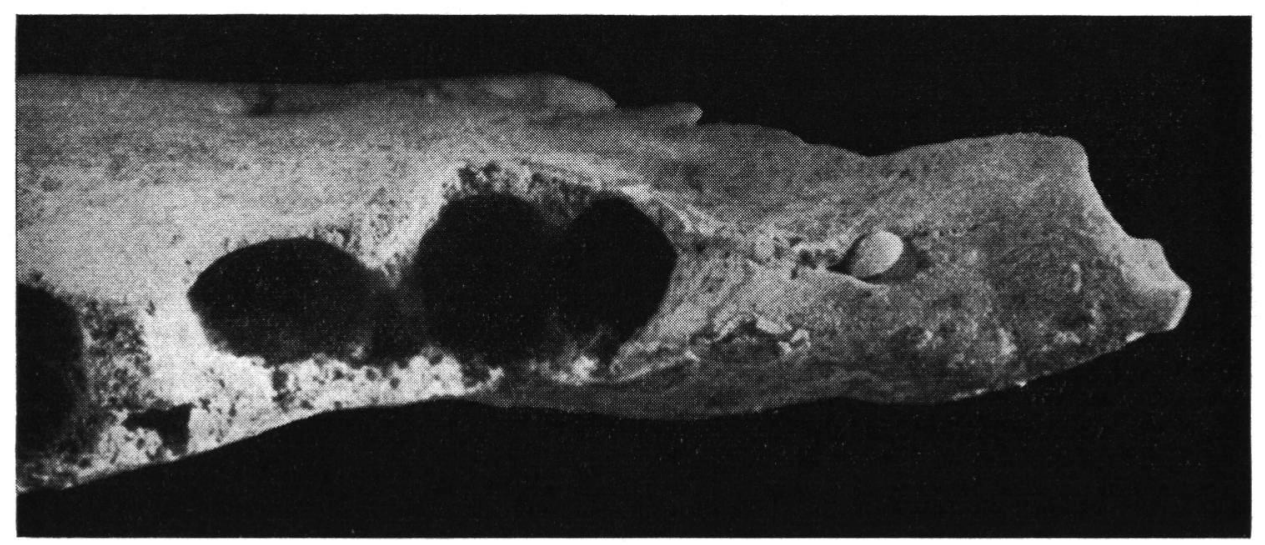

Fig. 17. U. spelaeus, mandibule sin. (partie mésiale) avec $\mathrm{d} 2(2 \mathrm{x})$.

10) Communiquée par M-elle E. Terzes à la séance mensuelle (mai, 1959) de l’Inst. de Spéol. 
L a mandibule sin. (Cl. 603) (Fig. 17), ci-dessus mentionnée, a sa $\mathrm{M}_{1}$ complètement apparue et la $\mathrm{a}^{2} \mathrm{M}_{2}$ avec une position encore oblique. La d2 implantée de biais, avec une couronne allongée, sans traces d'usure, rappelle selon sa morphologie générale la même dent décrite sur $1 \mathrm{a} \mathrm{m}$ a ndibule Cl. 563. La distance entre la $\mathrm{P}_{4}$ et la $\mathrm{C}$ est de $21.8 \mathrm{~mm}$; l'alvéole allongé de la $\mathrm{d} 2$ se situe à $13.5 \mathrm{~mm}$ après cette dernière; on peut observer aussi un vestige alvéolaire de la $\mathrm{d} 3$ collé à la paroi mésiale de l'alvéole de la $\mathrm{P}_{4}$.

Sur une autre ma n d ib u le ( $\mathrm{Cl}$. 608) presque de même âge, les alvéoles de la d2 et d3 sont présents, mais ils commencent à s'effacer.

Un cas plus particulier nous est offert par $1 \mathrm{a}$ m a ndibule sin. (Cl. 607) d'approximativement 16 mois; à la partie mésiale de la $\mathrm{P}_{4}$ complètement éclose, on voit un alvéole distinct, que nous avons attribué à la $\mathrm{d} 3$. Il est à remarquer le fait que dans ce cas l'alvéole de cette dent est bien séparé de celui de la $P_{1}$.

Aussi sur quelques mandibules adultes ( 3 sur 30 ) on peut observer un alvéole de dimensions réduites, situé à de variables distances de la $\mathrm{C}$, lequel d'après sa forme et position ne peut appartenir à la $\mathrm{P}_{4}$. Nous sommes portés à croire qu'il s'agit d'une persistance tardive de la d2.

En étudiant les diverses phases d'éruption de la dentition définitive et celles d'effacement des alvéoles des dents lactéales, il nous paraît possible que chez $U$. spelaeus la première dent à tomber soit la $\mathrm{d} 4$, suivie par la $\mathrm{d} 3$ et enfin la $\mathrm{d} 2$, qui peut persister assez. longtemps. Sur 24 mandibules où l'alvéole de la $\mathrm{d} 2$ s'est conservé, les dimensions qu'il comporte varient de 2 à $3.5 \mathrm{~mm}$ de longueur et le rapport long. C-P $4 /$ long. C-d2 est compris entre 1.61 et 2.71 .

Dans cette étude nous nous sommes bornés à décrire seulement la dentition jugale lactéale mandibulaire, parce que nous avons eu à notre disposition un matériel assez riche et varié. En ce qui concerne la dentition maxillaire, les quelques pièces que nous possédons, bien que fragmentaires, semblent indiquer les mêmes aspects observables sur la mandibule.

Selon les constatations déjà faites, les Ursidés ont dans leur première dentition 3 pièces jugales: d2, d3, et d4. Notre matériel nous a permis d'observer la réduction des jugales à deux: d 3 et d 4. La présence de la $P_{1}$ nous paraît fortuite, pouvant accompagner l'une ou l'autre des deux variantes, déjà exposées. La réduction des deux premières jugales et la persistance seulement de la $\mathrm{d} 4$, peut s'expliquer, croyons-nous, par un développement anormal, ayant en vue la rareté d'un tel matériel.

Nous avons constaté que la dent jugale antérieure (d2) présente une variabilité morphologique marquée, revêtant des formes assez différentes. L'absence des traces d'usure de cette dent et son emplacement dans une fossette, plus ou moins accentuée, de la mandibule, même aux pièces dénotant des animaux plus âgés (environ 16 mois), nous font croire que cette dent n'est pas fonctionnelle et se trouve en voie de réduction, témoin les exemplaires qui ne possèdent que la $\mathrm{d} 3$ et $\mathrm{d} 4$.

La dent jugale intermédiaire (d3) peut présenter deux racines bien distinctes qui se soudent en certains cas, ayant des traces de fusion plus ou moins évidentes; pour la plupart des cas on observe la d3 uniradiculée.

La dent jugale postérieure ( $\mathrm{d} 4)$ se manifeste comme une dent extrêmement variable, au schéma à 5 cuspides principales s'ajoutant un nombre plus ou moins élevé de petits tubercules secondaires et des formations aux dépens du bourrelet basilaire.

La mandibule la plưs jeune de notre matériel nous donne un aperçu sur l'ordre d'éclosion des jugales de la première dentition chez U. spelaeus, qui apparaissent comme suit: la $\mathrm{d} 4$ et la $\mathrm{d} 3$ presque en même temps, peut-être avec un léger décalage au profit de la première; après un court intervalle de temps se produit aussi l'éruption de la d2.

L'expulsion des jugales lactéales semble avoir lieu, aussi dans le sens disto-mésial; la $\mathrm{d} 2$ persiste en certaines occasions jusqu'à 16 mois et même plus. 
En essayant une interprétation du matériel exposé, il nous semble que la lignée évolutive d'Ursus spelaeus l'entrầne vers la réduction de la jugale antérieure, comme si le caractère phylétique s'inscrivait dans l'ontogénèse-cela s'est produit chez les Carnivores à formule simplifiée des prémolaires (Felidae) - mais ce caractère en voie de se fixer, n'a pu trouver sa réalisation avant l'extinction totale de l'espèce.

\section{Bibliographie}

Dubors, A. \& Stehlin, H. G.: La grotte de Cotencher, station moustérienne. - Mém. Soc. Paléont. Suisse, vol. 52/53, Bâle 1933.

Ehrenberg, K. in: Abel \& Kyrle, etc.: Die Drachenhöhle bei Mixnitz. - Speläologische Monographien, Bd. 7/8, 1931.

Friant, M.: Contribution à l'étude de la différenciation des dents jugales chez les Mammifères, 1933.

Kову, F.-Ed.: La dentition lactéale d’Ursus spelaeus. - Rev. Suisse Zool., T. 59, No. 27, 1952.

Kormos, Th.: Die Felsnische Pilisszánto. - Mitt. Jahrb. Kgl. ungar. Geol. Reichsanst. 23, Budapest 1916.

Morrt, M.: Über Stamm- und Artmerkmale der Bären. - Ung. Geol. Ges 21, II, Budapest 1934. - - Subalyuk-Höhle bei Cserépfalu. - Geol. Hung., Ser. Palaeont., fasc. 14, Budapest 1939.

Nordmann, A. v.: Palaeontologie Südrußlands. - Helsingfors 1858.

Owen, R.: A History of British Fossil Mammals and Birds. - London 1846.

Pohle, H.: Über den Zahnwechsel der Bären. - Zool. Anz. 55, Leipzig 1923.

Reynolds, S.: The Bears - A Monograph of the British Pleistocene Mammalia, vol. II, Part. II, London 1906.

Roska, M.: Traces de l'homme diluvien dans la caverne "Cholnoky" à Csoklovina. - Dolgozatok az Erdélyi nemzeti Muzéum Érem - ás Régiségtárábol, 3, 2, Kolozsvar 1912.

Schlosser, M.: Die Bären- oder Tischoferhöhle im Kaisertal bei Kufstein. - Abh. kgl. Bayer. Akad. d. Wiss., math.-phys. K1., 24, 2, München 1909.

Simionescu, I: Ursus spelaeus Blumb. din peştera Cioclovina (Hunedoara). - Ann. Acad. Rom.; Mem. Sect. St., Ser. III, t. 17, Mem. 7, Bucureşti 1942.

Soergel, W.: Der Bär von Süssenborn. - N.Jahrb. Min. Geol. u. Pal. 54BB, Abt. B., Stuttgart 1926.

Spahni, J.-Chr.: Méthode graphique appliquée à l'étude de la variabilité des dents de quelques Ursidés. - Eclogae Geol. Helv. 48, 2, Basel 1955.

Manuskr. eingeg. 2. 10. 1959.

Anschrift der Verf.: Dr. Constantin Rădulescu und Dr. Petre Samson, Speologisches Institut "E. Racoviţă“, Strada Dr. Câpşa 8 - R. Lenin, Bukarest, Kumänien. 